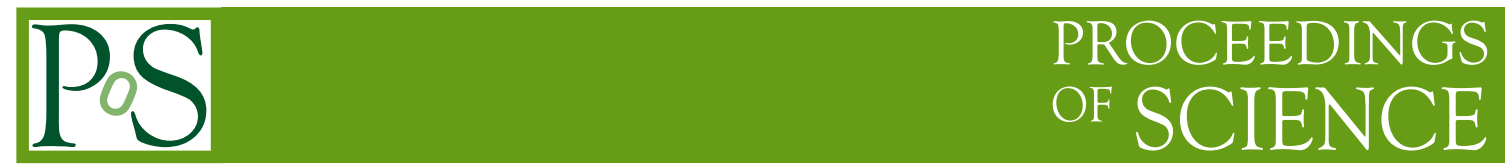

\title{
Searches for SUSY in events with two or more leptons in CMS
}

\author{
P. Martinez Ruiz Del Arbol* \\ On behalf of the CMS Collaboration \\ Eidgenössische Technische Hochschule Zürich (ETH Zurich), \\ E-mail: pablomecern.ch
}

\begin{abstract}
We present results of searches for SUSY production at CMS in events with multiple lepton production. These include final states with $\mathrm{Z}$ bosons decaying to lepton pairs, non-resonant sameand opposite-sign lepton pairs, and three or more isolated leptons. The results are used to exclude previously unexplored regions of the supersymmetric parameter space assuming R-parity conservation with the lightest supersymmetric particle being either a neutralino or gravitino.
\end{abstract}

36th International Conference on High Energy Physics,

July 4-11, 2012

Melbourne, Australia

${ }^{*}$ Speaker. 


\section{Introduction}

Supersymmetry (SUSY) is one of the most appealing extensions to the Standard Model, solving the hierarchy problem and providing dark matter candidates. The Compact Muon Solenoid (CMS) [1] collaboration has developed a complete program of SUSY searches covering a wide spectrum of final states. This document focuses on searches including two or more leptons, performed with data collected at $\sqrt{S}=7 \mathrm{TeV}$ and first results at $\sqrt{S}=8 \mathrm{TeV}$.

SUSY signatures usually involve the presence of large decay chains including high hadronic activity. In the case of R-parity-conserving SUSY, the production of invisible, stable particles (LSP) is also expected. Large jet multiplicity and high transverse missing energy $\left(\mathbb{E}_{T}\right)$ are therefore expected in any SUSY signature. The inclusion of leptons in the final state strongly suppresses the background and provides robust methods, based on data control regions, for estimating the remaining contribution.

The interpretation of the results is done in two different ways: in terms of the mSUGRA model, and using the so called simplified models (SMS)[2]. For the latter, a single decay chain is considered, and scanned for different values of the masses of the particles involved in the decay.

\section{Searches with two opposite sign leptons}

These searches cover final states containing jets, large $\mathbb{E}_{T}$ and two opposite-sign, same-flavor leptons. They are divided in on-peak searches aiming for final states with a $\mathrm{Z}$ boson decaying into leptons, and off-peak searches without the presence of a $Z$ peak. The main backgrounds in this final state are standard model $Z+$ jets and top pair production.
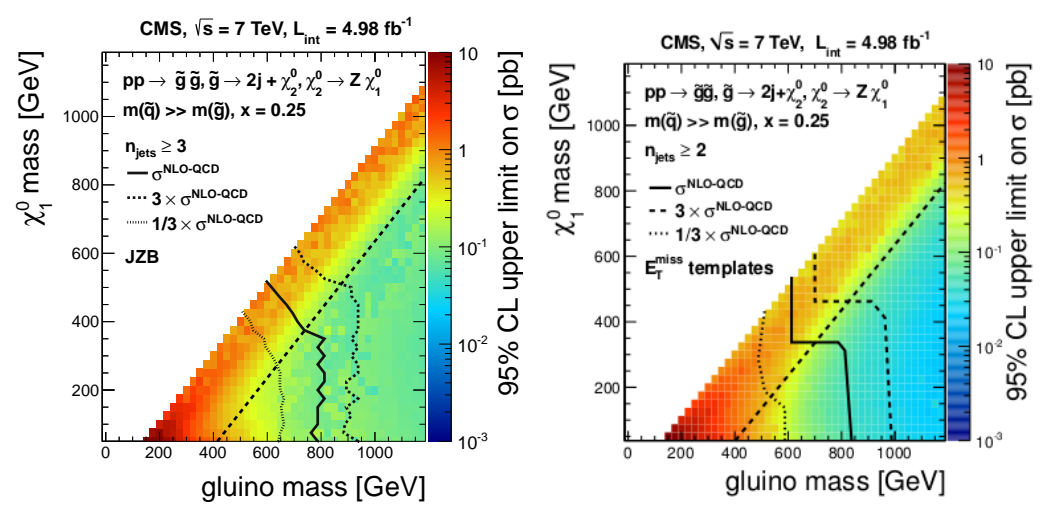

Figure 1: Upper limits on the masses of the gluino and the lightest neutralino for the JZB search (left) and the $\not_{T}$ templates search (right).

\subsection{On-peak search using the JZB (Jet-Z Balance) method at $\sqrt{S}=7 \mathrm{TeV}$}

This search looks for events containing jets, $\mathbb{E}_{T}$ and two same-flavor, opposite-sign leptons compatible with the $\mathrm{Z}$ mass. This search is sensitive to processes in which a gluino decays into quarks and a neutralino, and then into a $\mathrm{Z}$ boson and the lightest neutralino. 
The $Z+$ jets background is estimated using the Jet-Z Balance method (JZB)[3], based on the so called JZB variable, defined as the difference between the $P_{T}$ of the jet recoil and the $P_{T}$ of the dilepton system. In $Z+$ jets events, the $Z$ and the jets are balanced and events evenly populate the negative and positive JZB regions. The search region is defined for positive values of JZB while the negative region is used to estimate the $Z+$ jets contribution in the positive side.

The top pair background is estimated by using the lepton universality in this kind of decays which allows the use of the opposite-flavor lepton pairs to predict the contribution in the sameflavor channel.

No evidence of an excess is found in the $4.9 \mathrm{fb}^{-1}$ of data collected at $\sqrt{S}=7 \mathrm{TeV}$. Figure 1 (left) shows the upper limits set in terms of a SMS implementing the decay chain described above, parameterized for the masses of the gluino and the lightest neutralino.

\subsection{On-peak search using the MET templates method at $\sqrt{S}=7 \mathrm{TeV}$}

This search considers exactly for the same final state as the JZB search. The main backgrounds are the same: $Z+$ jets and top pair production. The latter is predicted using opposite-flavor pairs, as it was done in the JZB search, while for the former the MET templates method [3] is applied. This method exploits the fact that $\mathscr{E}_{T}$ in $Z+$ jets events is instrumental, and therefore can be predicted from events with a similar topology like $\gamma+$ jets or QCD.

The observed data is in good agreement with the data driven prediction, and upper limits are set on the same SMS as in the previous search (Fig. 1, right).
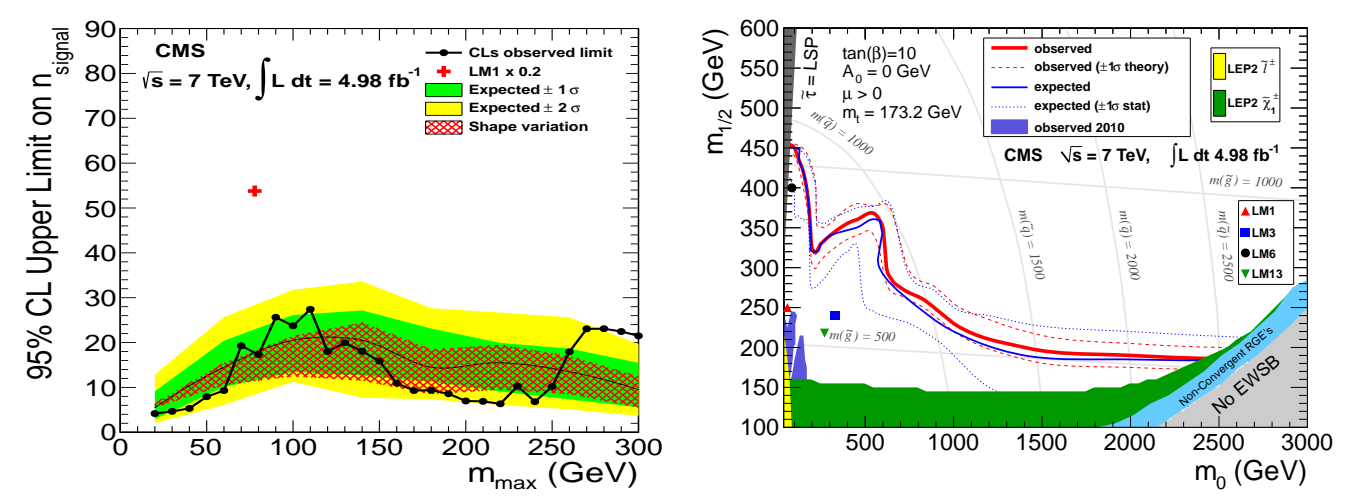

Figure 2: Upper limit on the number of yields for any given position of the edge structure (left). Upper limit on the mSUGRA plane using the generic off-peak search (right).

\subsection{Off-peak search using the Edge method at $\sqrt{S}=7 \mathrm{TeV}$}

The final state considered in this search contains jets, $\mathbb{E}_{T}$ and a pair of opposite-sign, sameflavor leptons, without restriction on their invariant mass. This final state is realized in decay chains with slepton productions in three body decays, including two leptons and the LSP. This configuration produces a kinematical edge in the invariant mass distribution. The main backgrounds for this search are again $Z+$ jets and top pair production. The edge method[4] performs a fit to the invariant mass distribution looking for an edge. The $Z+$ jets component is fitted as well, using a $Z$-shape 
model, while the top pair production contribution is fitted in combination with the opposite flavor invariant mass. The number of events under the fitted edge is compatible with 0 within uncertainty. Upper limits are set on the yields for any given position of the edge in the mass range (Fig. 2, left).

\subsection{Generic off-peak search at $\sqrt{S}=7 \mathrm{TeV}$}

The generic off-peak search [4] looks for an excess of events in the same final state as the Edge method. The $Z+$ jets background is reduced by applying high $\mathbb{E}_{T}$ and $H_{T}$ (sum of the $P_{T}$ of the jets) cuts, and by rejecting events in which the mass of the dilepton system is close to the $\mathrm{Z}$ mass. The $Z_{T}$ in events with top pair production is predicted using the similarity between the spectrum of the pair of neutrinos and the pair of leptons.

The predicted backgrounds are in good agreement with the observation and upper limits are calculated in the context of mSUGRA (Fig. 2, right).

\section{Searches with two same sign leptons}

Several searches in CMS look for final states including large $H_{T}$ and $\mathscr{E}_{T}$, jets and a pair of same sign leptons. These searches are sensitive to decay chains involving strong or electroweak production with sbottoms and stops in the intermediate steps decaying into top and $\mathrm{W}$ particles.

The strength of this final state is related to the relatively small amount of Standard Model background. There are three different sources: events with non-prompt leptons coming from QCD, $\mathrm{W}+$ jets, or heavy flavor leptons in top pair production, $Z+$ jets events with charge misassignment in one of the leptons, and rare Standard Model processes.

Contributions from non-prompt leptons are estimated by relaxing the isolation cuts in data control regions rich in non-prompt leptons and extrapolating into the signal region. The charge misassignment probability is also estimated in a control region dominated by $Z+$ jets events, while rare Standard Model processes are estimated from Monte Carlo.

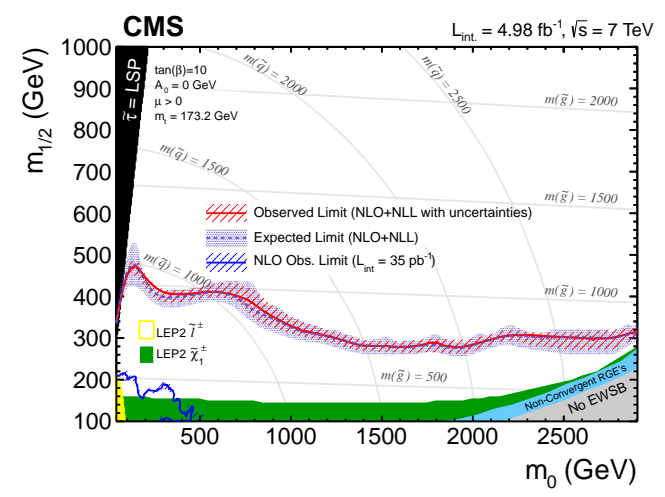

Figure 3: Upper limits on the mSUGRA plane with the same sign search at $\sqrt{S}=7 \mathrm{TeV}$

\subsection{Two same sign leptons search at $\sqrt{S}=7 \mathrm{TeV}$}

This search[5] defines several signal regions in terms of $\mathbb{E}_{T}$ and $H_{T}$. The main rare Standard Model processes contributing as background are diboson production such as WW and WZ. Figure 
3 shows the results obtained for a total integrated luminosity of $5 \mathrm{fb}^{-1}$ collected at $\sqrt{S}=7 \mathrm{TeV}$. The predicted background is in good agreement with the observation in all the different signal regions. Exclusion limits are set in the context of mSUGRA.
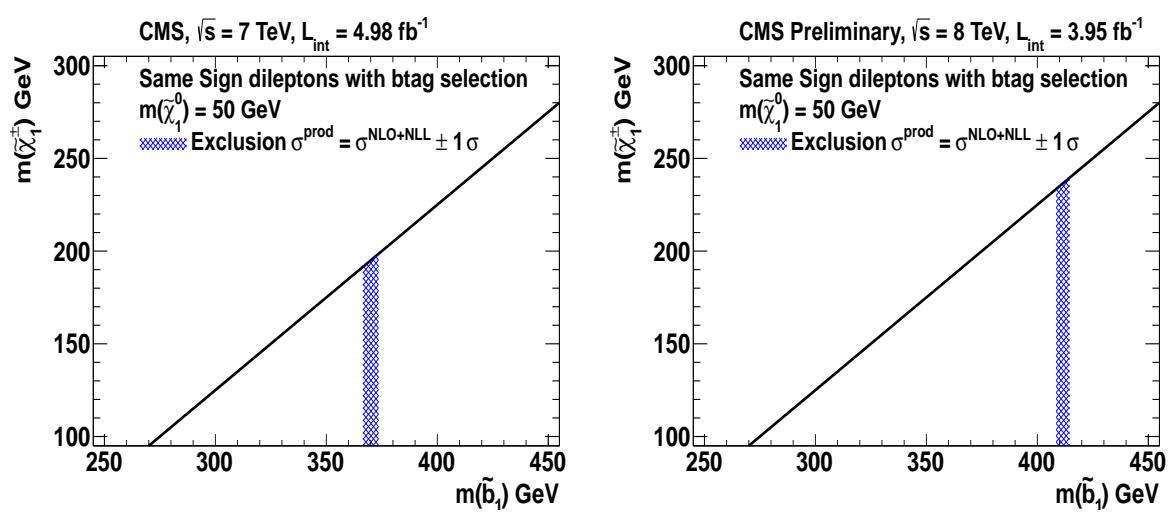

Figure 4: Upper limits on the masses of the sbottom and the charginos for an SMS with direct sbottom production using data collected at $\sqrt{S}=7 \mathrm{TeV}$ (left) and $\sqrt{S}=8 \mathrm{TeV}$ (right).

\subsection{Two same sign leptons and b-jets search at $\sqrt{S}=7$ and $8 \mathrm{TeV}$}

The final state considered in this search $[6,7]$ requires, on top of the same sign lepton pair, at least one b-jet in the event. The main rare processes contributing to this final state are ttW and $\mathrm{ttZ}$ production. The results are interpreted in terms of SMSs implementing decays of gluinos into $t+\bar{t}$ pairs and the lightest neutralino, or into top-stop pairs, decaying into top and the lightest neutralino.

The background prediction is in good agreement with the observation, and upper limits are set in the context of several simplified models. Figure 4 shows upper limits on the mass of the sbottom and chargino for the search at $\sqrt{S}=7 \mathrm{TeV}$ (left) and the search at $\sqrt{S}=8 \mathrm{TeV}$ (right).

\section{Searches with more than two leptons at $\sqrt{S}=7 \mathrm{TeV}$}

This search[8] looks for a final state with three or more leptons. The aim is to have sensitivity to decay chains involving direct chargino or neutralino production, decaying in sleptons or $\mathrm{W}+\mathrm{Z}$. The main backgrounds for these searches are non-prompt leptons in $Z+$ jets events, backgrounds from Standard Model processes such as WW, WZ or top pair production, and $Z+$ jets events with final state radiation and converted photons.

The rate of non-prompt leptons is calculated by relaxing the isolation cuts and applied to the signal region. Irreducible backgrounds are taken from Monte Carlo, while the rate of converted photons in final state radiation is calculated from a control sample rich in $Z+$ jets events.

The results for this search are shown in figure 5. No evidence of an excess over the predicted background is found. Upper limits on the masses of the neutralinos and charginos are set in the context of SMS implementing the decays described above. 


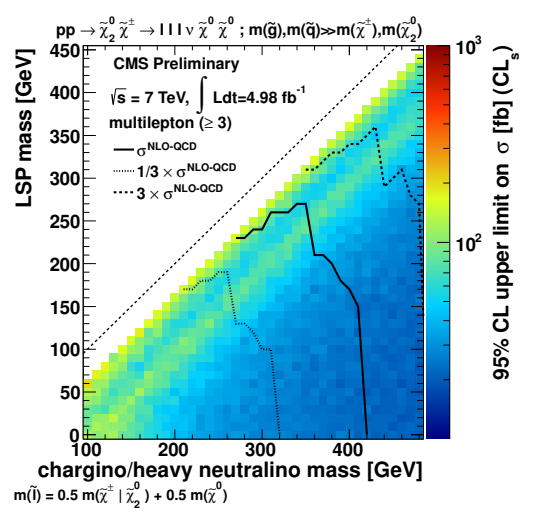

Figure 5: Upper limits on the masses of the chargino or the heavy neutralino and the lightest neutralino in an SMS with direct chargino and neutralino production.

\section{Conclusions}

Several searches for SUSY in events with two or more leptons have been performed using data collected by the CMS experiment at $\sqrt{S}=7 \mathrm{TeV}$ and $\sqrt{S}=8 \mathrm{TeV}$. These include searches with two opposite sign leptons inside and outside the $\mathrm{Z}$ mass, searches with two same sign leptons, requiring also one b-tagged jet, and searches with three or more leptons. In all the cases the observation is in good agreement with the data driven predictions, and upper limits are set in terms of mSUGRA and simplified models.

\section{References}

[1] The CMS Collaboration, "The CMS experiment at the CERN LHC", JINST 3 S08004(2008), doi:10.1088/1748-0221/3/08/S08

[2] The CMS Collaboration, "Interpretation of Searches for Supersymmetry", CMS-PAS-SUS-11-016

[3] The CMS Collaboration, "Search for physics beyond the standard model in events with a Z boson, jets, and missing transverse energy in pp collisions at $\sqrt{s}=7 \mathrm{TeV}$ ", Physics Letters B, 716, 2, 260-284, doi:10.1016/j.physletb.2012.08.026

[4] The CMS Collaboration, "Search for new physics in events with opposite-sign leptons, jets, and missing transverse energy in pp collisions at $\sqrt{s}=7 \mathrm{TeV} "$, CMS-PAS-SUS-11-011

[5] The CMS Collaboration, "Search for New Physics with Same-Sign Isolated Dilepton Events with Jets and Missing Transverse Energy", Phys. Rev. Lett., 109, 7, 071803, 16, doi:10.1103/PhysRevLett.109.071803

[6] The CMS Collaboration, "Search for new physics in events with same-sign dileptons and b-tagged jets in pp collisions at $\sqrt{s}=7 \mathrm{TeV}$ ", Journal of High Energy Physics, 2012, 8, doi:10.1007/JHEP08(2012)110

[7] The CMS Collaboration, "Search for Supersymmetry in events with same-sign dileptons", CMS-PAS-SUS-12-017

[8] The CMS Collaboration, "Search for anomalous production of multilepton events in pp collisions at $\sqrt{s}=7 \mathrm{TeV}$ ", Journal of High Energy Physics, 2012, 6, doi:10.1007/JHEP06(2012)169 